Características reproductivas, sanitarias y de manejo en establecimientos lecheros del Uruguay

\title{
Reproductive, health and management characteristics in dairy herds in Uruguay
}

Alejandra Suanes ${ }^{1}$ 0000-0002-1513-7734
Valentina Macchi ${ }^{1}$ 0000-0002-1836-852
Federico Fernández ${ }^{2}$ 0000-0001-9032-9798

'División de Laboratorios Veterinarios “Miguel C. Rubino”, Ministerio de Ganadería, Agricultura y Pesca. Ruta 8 Brig. Gral. J. A.

Lavalleja Km. 17.500. Montevideo, Uruguay. Autor para correspondencia: asuanes@mgap.gub.uy

${ }^{2}$ Dirección General de Servicios Ganaderos, Ministerio de Ganadería, Agricultura y Pesca, Montevideo, Uruguay

${ }^{3}$ Facultad de Veterinaria, Universidad de la República, Montevideo, Uruguay

Veterinaria (Montevideo) Volumen 57

№ 215 (2021 Ene - Jun) e20215721503

DOI:10.29155/VET.57.215.3

(c) EY-NC

Recibido: $12 / 05 / 2020$

\section{Resumen}

Los problemas reproductivos y sanitarios representan una de las limitantes de mayor impacto productivo en los rodeos lecheros del Uruguay. Las alteraciones en los índices reproductivos pueden deberse a una amplia diversidad de causas, las cuales exigen un correcto diagnóstico. Actualmente los antecedentes de encuestas en el rubro lechero son proporcionados por la Oficina de Estadísticas Agropecuarias y el Instituto Nacional de Leche. El objetivo de este estudio fue caracterizar las prácticas de manejo, reproductivas y sanitarias en los establecimientos lecheros del Uruguay. Se diseñó un estudio transversal de los establecimientos lecheros del Uruguay. Se creó una encuesta la cual fue realizada en el año 2015. Fueron seleccionados 107 predios lecheros de acuerdo al tamaño de población bovina a partir de un muestreo aleatorio estratificado. Las preguntas se enfocaron en características generales del predio, estrategias y características sanitarias, y estrategias y características reproductivas. La tasa de respuesta fue del 95,3\%. De los predios encuestados, el 95,6 $\pm 1,8 \%$ tenía asesoramiento veterinario. La técnica de inseminación artificial se utilizó en el 83,8 $\pm 5,2$ $\%$. Un 59,9 $\pm 5,5 \%$ de los predios realizaban diagnóstico de gestación. La tasa de preñez estimada fue del 79,5 $\pm 1,5 \%$. La evaluación de los toros previo al servicio se realizó en el 49,0 $\pm 7,6 \%$ de los tambos. La principal causa de refugo fue por dentición. La utilización de vacunas como medida preventiva para enfermedades reproductivas se utilizó en el 56,6 $\pm 5,4 \%$ de los productores lecheros. El 58,7 $\pm 6,7 \%$ de los encuestados declaró no tener ningún antecedente de enfermedades reproductivas. La media de abortos vistos anualmente fue de 4,4 $\pm 0,6$. Las tasas de preñez no pudo asociarse a aspectos reproductivos y/o sanitarios. Se pudo determinar que el aspecto demográfico cumple un rol significativo en la elección de los aspectos reproductivos y de manejo en los establecimientos lecheros del Uruguay. Palabras clave: Encuesta, tambos, bovinos, indicadores reproductivos.

\section{Abstract}

Ones of the most important limitations on dairy herd of Uruguay are the reproductive and health problems. Alterations on reproductive rates may be due to a wide variety of causes, which require a correct diagnosis. Currently in Uruguay, histories of surveys in dairy sector were provided by the "Oficina de Estadísticas Agropecuarias" and the "Instituto Nacional de Leche". The aim of this study was to characterize the management, reproductive and health practices in dairy farms in Uruguay. A cross-sectional study was carried out in 2015. Dairy herds were stratified by size and 107 herds were randomly selected throughout all the Uruguayan territory. A survey was made on these herds; the questions were focused on general characteristics of the farms, strategies and health characteristics, and strategies and reproductive characteristics. The response rate of the survey was $95.3 \%$. Of the surveyed farms, $95.6 \pm 1.8 \%$ had veterinary service. The artificial insemination technique was used in $83.8 \pm$ $5.2 \%$. Fifty-nine percent of the farms made a pregnancy diagnosis. The estimated pregnancy rate was $79.5 \pm 1.5 \%$. The pre-service evaluation of the bulls was carried out in $49.0 \pm 7.6 \%$ of the dairy farms. The main cause of culling was due to teething. Use of vaccines as a preventive measure for reproductive diseases was $56.6 \pm 5.4 \%$ of the dairy farms. Fifty-eight percent of those surveyed had no history of reproductive diseases. The mean of abortions seen annually was $4.4 \pm 0.6$. Pregnancy rates could not be associated with reproductive and/or health aspects. It was possible to determine that the demographic aspect is a key role in the choice of reproductive and management aspects in dairy farms in Uruguay.

Keywords: Survey, dairy, cattle, reproductive indicators 


\section{Introducción}

La industria láctea uruguaya es uno de los sectores más importantes del país, participando en el valor de exportaciones de la economía nacional con U\$ 650 millones (INALE, 2019). Uruguay es el tercer exportador mundial de leche en polvo entera, vendiendo al exterior el $70 \%$ de lo producido a nivel local. Los establecimientos lecheros se distribuyen en su mayoría en la cuenca sur y litoral oeste del país. En la actualidad existe una tendencia general a la reducción en el número de predios y un aumento de predios con mayor superficie y/o vacas en ordeñe, por lo que la producción de leche tiende a concentrarse (OPYPA, 2019). Las exigencias del mercado internacional requieren una permanente mejora del sector para el mantenimiento de la competitividad. Los problemas reproductivos y sanitarios representan una de las limitantes de mayor impacto en los rodeos lecheros. Las alteraciones en los índices reproductivos pueden deberse a una amplia diversidad de causas, las cuales exigen un correcto diagnóstico para poder realizar una buena evaluación de la performance reproductiva del sector.

Internacionalmente existen ejemplos exitosos en el desarrollo de sistemas de monitoreo en salud animal. Un buen ejemplo es el "National Animal Health Monitoring System" (NAHMS) del "United State Department of Agriculture" (USDA) el cual aporta información de buena calidad que representa la población objetivo. Comparable con el NAHMS, también se puede citar el "National Dairy Study" de Canadá (NDS) (Bauman et al., 2016). Basado en una gran encuesta, el NDS generó datos sobre la demografía del rebaño, la bioseguridad, el cuidado y el bienestar de los animales, la salud de los terneros, el manejo reproductivo, y calidad de la leche (Renaud et al., 2017). Estos autores evidenciaron que un adecuado empleo de los indicadores antes mencionados hace posible la toma de decisiones para un correcto manejo reproductivo basados en la búsqueda de metas concretas y posibles (Bauman et al., 2016; Belage et al., 2017).

Los productores enfrentan continuamente diferentes desafíos a medida que los mismo pasan de manejos tradicionales a manejos que involucran mayor tecnología, esta evolución es interesante monitorearla. Una posible metodología es a través de la recolección de datos a partir de encuestas, y a través del análisis de la misma realizar una descripción actualizada del sector. En Uruguay ya existen antecedentes de encuestas en lechería, las mismas son proporcionadas por la Oficina de Estadísticas Agropecuarias (DIEA) y el Instituto Nacional de Leche (INALE). Además, existe a nivel nacional antecedentes de aportes de encuestas realizados por profesionales del sector técnico académico (Ibarra et al., 2002; Zaffaroni et al., 2003; Lemaire et al., 2011; Pereira et al., 2017). Es importante el relevamiento y la actualización continua de datos nacionales de los diferentes rubros agropecuarios. Es por ello, que con la finalidad de generar un aporte al sector, este trabajo tiene como objetivo principal caracterizar, a través de un muestreo estadísticamente representativo, las prácticas de manejo, reproductivo y sanitario en los establecimientos lecheros del Uruguay.

\section{Materiales y Métodos}

\section{Diseño de muestreo}

Se realizó un estudio transversal de los establecimientos lecheros del Uruguay. Se creó una encuesta la cual fue realizada del 1 de agosto al 31 de diciembre de 2015. Fueron seleccionados 107 predios lecheros a partir de un muestreo aleatorio estratificado distribuidos en todo el territorio uruguayo. Para el diseño del marco de muestreo se solicitó a la División de Contralor de Semovientes (DICOSE) la declaración jurada del año 2014 la cual contenía todos los bovinos declarados por predio con identificación individual. Se incluyeron 695.811 bovinos lecheros, pertenecientes a 3.042 tambos (DIEA y MGAP, 2014). Se definieron como unidades primarias de muestreo los predios lecheros los cuales fueron distribuidos en tres estratos según el tamaño de la población bovina (Tabla 1); dentro de cada estrato se seleccionó un número similar de predios, independientemente de la cantidad de predios que hubiera por estrato.

Tabla 1. Distribución de bovinos, vacas adultas y predios lecheros del Uruguay según estrato de población bovina (año 2015).

\begin{tabular}{lllll}
\hline Estrato $^{\mathbf{A}}$ & Bovinos & Vacas & Predios & $\boldsymbol{n}_{(\mathbf{m u e s t r a})^{\mathbf{B}}}$ \\
\hline $\mathbf{1 - 5 0}$ bovinos & 19.438 & 13.491 & 626 & 35 \\
$\mathbf{5 1 - 2 5 0}$ bovinos & 206.447 & 143.548 & 1.670 & 36 \\
$>\mathbf{2 5 0}$ bovinos & 469.926 & 317.170 & 746 & 36 \\
Total & $\mathbf{6 9 5 . 8 1 1}$ & $\mathbf{4 7 4 . 2 0 9}$ & $\mathbf{3 . 0 4 2}$ & $\mathbf{1 0 7}$ \\
\hline
\end{tabular}

${ }^{\mathrm{A}}$ Los estratos se determinaron según la población de bovinos en cada predio.

${ }^{\mathrm{B}}$ Cantidad de predios seleccionados para muestrear por estratos. 
La encuesta fue diseñada por un equipo multidisciplinario perteneciente a la Dirección General de Servicios Ganaderos (DGSG) y Facultad de Veterinaria. El grupo asesor modificó, refinó y validó previamente el cuestionario previo a su ejecución. En los establecimientos lecheros los veterinarios oficiales de Ministerio de Ganadería Agricultura y Pesca (MGAP) fueron los responsables de realizar la encuesta a los productores o personal autorizado al momento de la visita al establecimiento. Se utilizaron tabletas electrónicas para recabar la información y la misma se almacenó en planillas usando el software Microsoft Excel (Microsoft Corporation, Redmond, WA). La encuesta consistió en 4 preguntas abiertas y 31 preguntas cerradas (Anexo I). Las preguntas se enfocaron en características generales del predio, estrategias y características sanitarias, y estrategias y características reproductivas (Tabla 2 ). mismo la probabilidad de selección de los predios fue considerada para ponderar los análisis y proyectar las estimaciones a la población general. Las asociaciones simples se probaron usando pruebas de Chi-cuadrado con una corrección de segundo orden (Rao y Scott, 1984) con un nivel de significancia de $\mathrm{p}<0,05$. Se estimó la media y error estándar de forma general y para cada estrato en cada una de las variables.

Tabla 2. Resumen de encuesta realizada a productores lecheros del Uruguay durante el segundo semestre del año 2015.

\begin{tabular}{ll}
\hline Sección & Preguntas \\
\hline \multirow{2}{*}{ Características generales del predio } & Tipo de entrevistado, presencia de asesoramiento veterinario, tipo de asesora- \\
& miento veterinario (eventual o permanente). \\
& Inseminación artificial: a celo visto, tiempo fijo, repaso con toros. Servicio con- \\
& tinuo.
\end{tabular}

Estrategias reproductivas

Uso de tecnologías: manejo por CC, diagnóstico de gestación, DEP, edad de entore a los dos años, ecografía (diagnóstico de gestación, actividad ovárica).

Características reproductivas $\quad$ Número de hembras servidas en el último año, porcentaje de preñez.

Revisión de toros: condición física, sanitaria, capacidad de servicio, evaluación de calidad seminal.

Uso de toros: compartidos, compra en remate, de descarte, vacunados, jóvenes,

Estrategias sanitarias porcentaje de mayores de cuatro años.

Causa de refugo de hembras: sanitaria, dentición, primera falla o segunda falla reproductiva.

Vacunación contra enfermedades reproductivas

Número de abortos vistos el año anterior, causa presumible de aborto, variación del porcentaje de aborto con respecto al año anterior, nacimiento de terneros dé-

Características sanitarias biles o muertos, existencia de repetición de celos o ciclos largos, antecedentes de enfermedades reproductivas.

$\mathrm{DEP}=$ Diferencia esperada de progenie. $\mathrm{CC}=$ Condición corporal

\section{Análisis estadístico}

Los datos se exportaron desde Microsoft Excel al software estadístico STATA/IC 15 (StataCorp. 2017. Stata Statistical Software: Release 15. College Station, TX: StataCorp LLC) para su depuración, validación y análisis. El análisis estadístico se realizó teniendo en cuenta el diseño del muestreo, a través de las rutinas para muestreos complejos del software estadístico STATA/IC 15. Se generaron distribuciones y estadísticas descriptivas para cada una de las variables de la encuesta. Así

\section{Resultados}

\section{Muestreo y características de los encuestados}

Se analizaron las encuestas de 102 predios lecheros con una amplitud de 1 a 1179 vacas. La media de vacas adultas dentro de cada estrato fue para el caso del estrato de 1 a 50 bovino $23,7 \pm 14,6$, para el de 51 a 250 bovinos $70,4 \pm 37,3$, y para el estrato con más de 250 bovinos 303,5 $\pm 209,9$. 
La tasa de respuesta de la encuesta fue del 95,3\%. El $65,1 \pm 5,4 \%$ de los entrevistados que realizaron la encuesta eran propietarios del predio, seguidos por familiares $(19,0 \pm 4,6 \%)$, encargados o veterinarios $(10,1 \pm 3,3 \%)$, capataces $(2,8 \pm 1,4 \%)$ y otros $(3,0 \pm 1,9 \%)$. No detectándose diferencias significativas entre los estratos de población bovina.

\section{Características generales de los predios}

De los predios encuestados, el 95,6 $\pm 1,8 \%$ tenía asesoramiento veterinario, de los cuales el 49,2 $\pm 5,5 \%$ era de tipo permanente mientras que el $49,1 \pm 5,4 \%$ era eventual. Existió una asociación positiva entre el tipo de asesoramiento y el tamaño del predio $(\mathrm{p}<0,01)$. Los predios de menor tamaño suelen contar con asesoramiento veterinario del tipo eventual, mientras que los veterinarios permanentes fueron más frecuentes en predios de mayor tamaño (Figura 1).

\section{Estrategias reproductivas}

La técnica de inseminación artificial (IA) fue utilizada por el $83,8 \pm 5,2 \%$ de los productores, no existiendo diferencias significativas entre estratos. Dentro de este porcentaje, el 72,0 $\pm 6,2 \%$ realizaba la IA a celo visto. La inseminación artificial a tiempo fijo (IATF) era utilizada por el $15,9 \pm 4,0 \%$ de los predios, habiendo diferencias significativas entre estratos (Tabla 3). Así mismo, el 10,0 $\pm 4,3 \%$ realizaban tanto IA a celo visto como IATF, esto era realizado únicamente por el estrato $>250$ bovinos. El 36,1 $\pm 6,5 \%$ de los establecimientos realizaba repaso con toro luego de la IA. No hubo asociación entre la tasa de preñez y el empleo de estas técnicas.

En cuanto al empleo de otras tecnologías reproductivas el 20,2 $\pm 4,2 \%$ utilizaban manejo diferencial según condición corporal de las vacas. Mientras que el uso de diferencia esperada de progenie (DEP) era empleado por un 13,1 $\pm 3,5 \%$ de los productores, utilizándose en mayor medida por los predios más grandes (Tabla 3).

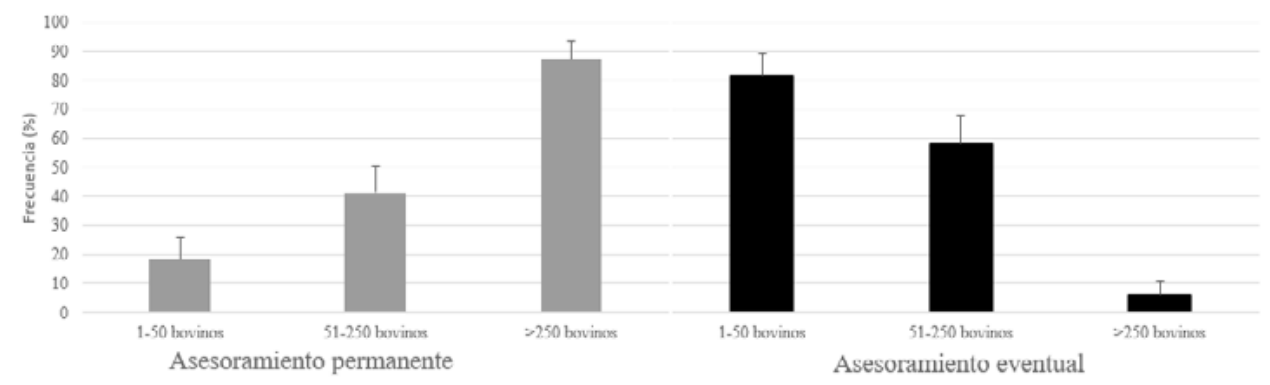

Figura 1: Porcentaje de predios lecheros que cuentan con asesoramiento veterinario permanente o eventual según los diferentes estratos de población bovina $(\mathrm{p}<0,01)$.

Tabla 3. Utilización de estrategias reproductivas de acuerdo a los estratos de población bovina en predios lecheros del Uruguay (Media \pm Error Estándar).

\begin{tabular}{lcccc}
\hline & $1-50$ bovinos $(\%)$ & $50-250$ bovinos $(\%)$ & >250 bovinos $(\%)$ & valor- $p$ \\
\cline { 2 - 5 } IA & $80,0( \pm 10,1)$ & $80,0( \pm 9,2)$ & $90,6( \pm 5,2)$ & 0,50 \\
Celo visto & $73,3( \pm 11,8)$ & $65,9( \pm 10,9)$ & $81,3( \pm 7,0)$ & 0,38 \\
IATF & $0^{\mathrm{a}}$ & $5,0( \pm 5,0)^{\mathrm{a}}$ & $37,5( \pm 8,7)^{\mathrm{b}}$ & $<0,01$ \\
Repaso toros & $13,3( \pm 9,1)$ & $35,0( \pm 10,9)$ & $46,9( \pm 9,0)$ & 0,18 \\
Manejo por CC & $20,0( \pm 8,2)^{\mathrm{ab}}$ & $10,7( \pm 6,0)^{\mathrm{a}}$ & $37,1( \pm 8,3)^{\mathrm{b}}$ & 0,04 \\
DEP & $0^{\mathrm{a}}$ & $7,1( \pm 5,0)^{\mathrm{ab}}$ & $32,4( \pm 8,1)^{\mathrm{b}}$ & $<0,01$ \\
Edad de entore a los dos años & $64,0( \pm 9,8)$ & $60,7( \pm 9,4)$ & $65,7( \pm 8,1)$ & 0,89 \\
Servicio continuo & $73,6( \pm 10,3)^{\mathrm{a}}$ & $76,0( \pm 8,7)^{\mathrm{a}}$ & $36,6( \pm 10,5)^{\mathrm{b}}$ & 0,01 \\
\hline
\end{tabular}

IA = Inseminación artificial. IATF $=$ Inseminación artificial a tiempo fijo. $\mathrm{CC}=$ Condición corporal. $\mathrm{DEP}=\mathrm{Diferencia}$ esperada de progenie.

Letras iguales indican $\mathrm{p} \geq 0,05$ y letras diferentes indican diferencia estadística entre estratos $(\mathrm{p}<0,05)$ 


\section{Características reproductivas}

En la Fig. 2 se observa la asociación entre la utilización del diagnóstico de gestación y el tamaño de los predios leche$\operatorname{ros}(\mathrm{p}<0,01)$. De acuerdo a las respuestas de los encuestados, un $59,9 \pm 5,5 \%$ de los predios realizaba diagnóstico de gestación. Solo el 13,7 $\pm 3,6 \%$ de los que contestaron realizar diagnóstico de gestación realizaban ecografía, siendo claramente mayor el uso de esta tecnología para el caso de los predios con más de 250 bovinos (Fig. 2; p=0,01). El 17,6 $\pm 4,1 \%$ de los productores realizaban ecografía para la evaluación de la actividad ovárica, los predios más grandes presentaron una tendencia a realizarlo en mayor proporción. No se encontró asociación de esta medida con la tasa de preñez. Por otro lado, el $62,8 \pm 5,8 \%$ de los productores entoraban las vaquillonas con 2 años, no encontrándose diferencias entre estratos (Tabla 3).

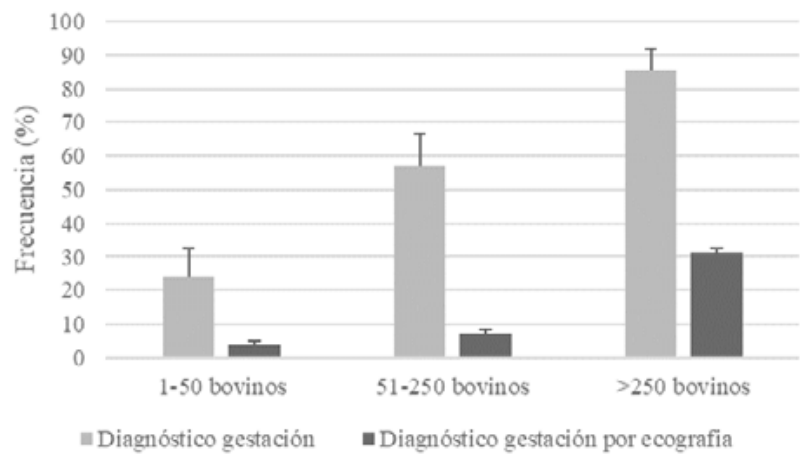

Figura 2: Porcentaje de predios lecheros que realizan diagnóstico de gestación $(\mathrm{p}<0,01)$ y utilización de ecografía $(\mathrm{p}=0,01)$ para dicho diagnóstico según los diferentes estratos de población bovina.

El porcentaje de preñez en aquellos predios que realizan diagnóstico de gestación se estimó en $79,7 \pm 2,0 \%$, no encontrándose asociación entre estratos, ni con el asesoramiento profesional en el predio.

El $66,35 \pm 5,9 \%$ de los tambos realizaban el servicio de forma continua durante el año, encontrándose diferencias significativas entre estratos (Tabla 3; $\mathrm{p}=0,01$ ). El total de vacas $\mathrm{y}$ vaquillonas servidas el último año se detalla en la Tabla 4 , y como era de esperarse a medida que el tamaño del predio aumenta también lo hace la cantidad de hembras servidas.

Tabla 4. Promedio de vacas y vaquillonas servidas en el año 2014 en predios lecheros del Uruguay según estrato de población bovina.

\begin{tabular}{lll}
\hline Estratos & Media & Error estándar \\
\hline 1-50 bovinos & 29,7 & 4,7 \\
$51-250$ bovinos & 63,7 & 5,8 \\
$>250$ bovinos & 284,3 & 32,4 \\
Promedio general & 111,9 & 8,8 \\
\hline
\end{tabular}

\section{Estrategias sanitarias}

La evaluación de los toros previo al servicio se realizaba en el 49,0 $\pm 7,6 \%$ de los predios, siendo la condición física $(44,2 \pm 7,6 \%)$ y sanitaria $(39,7 \pm 7,1 \%)$ de los toros la más frecuentemente evaluada. Por otro lado, la capacidad de servicio era realizada sólo por el $10,0 \pm 4,7 \%$ de los tambos; y la evaluación de la calidad seminal por el $11,1 \pm 4,3 \%$. No se encontraron diferencias significativas entre estratos.

De acuerdo al origen de los toros utilizados durante la época de servicio, se reportó que el $8,7 \pm 3,3 \%$ utilizaba toros compartidos, mientras que el 21,3 $\pm 5,1 \%$ de los predios los adquirían en remates o ferias. En los establecimientos existía un $11,9 \pm 3,8 \%$ de toros mayores a 4 años, no hubo diferencias significativas entre estratos. Los toros de descarte eran utilizados por el 1,5 $\pm 1,1 \%$ de los tambos, habiendo diferencias entre estratos ( $p<0,01)$, los únicos que los utilizaban eran los tambos que pertenecían al estrato de 1-50 bovinos siendo el porcentaje de $8,3 \pm 5,8 \%$. El porcentaje de encuestados que reportó utilizar toros jóvenes en sus rodeos era del $52,7 \pm 6,1 \%$, mientras que la vacunación de toros previo al servicio era empleada por el $46,5 \pm 6,0 \%$

La distribución de causas de refugo en la población de vacas se muestra en la Figura 3, siendo la principal causa por dentición. Únicamente en los refugos por problemas sanitarios se reportaron diferencias significativas entre estratos. Los estratos con 1-50 bovinos refugan por esta causa en menor medida $(21,4 \pm 7,9 \%)$ que aquellos estratos con $>250$ bovinos $(58,6$ $\pm 9,3 \%)(\mathrm{p}=0,04)$.

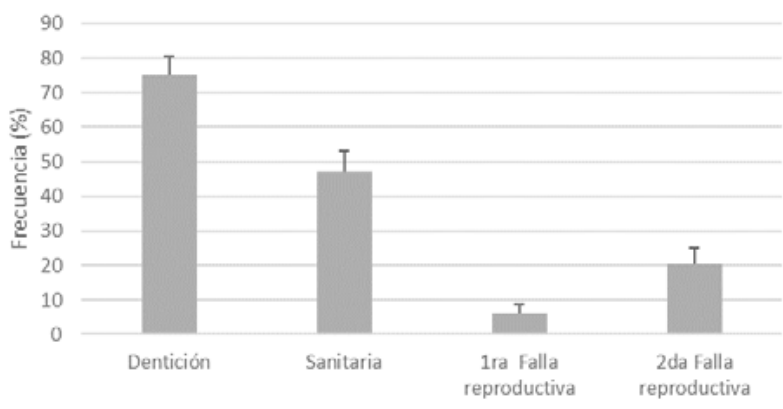

Figura 3: Causas de refugo de la población de vacas de cría en predios lecheros del Uruguay.

La utilización de vacunas como medida preventiva para enfermedades reproductivas según los encuestados era utilizada por el 56,6 $\pm 5,4 \%$ de los productores lecheros. Esta variable se asoció con el tamaño del predio $(\mathrm{p}=0,03)$ y con el tipo de asesoramiento veterinario $(p<0,01)$, pero no con la presencia de antecedentes de enfermedades reproductivas (AER). Las vacunas eran más utilizadas por los estratos de $>250$ bovinos $(93,8$ $\pm 4,3 \%$ ) y en menor medida por los estratos de 1-50 bovinos $(36,7 \pm 4,3 \%)$. Como era esperable, esta herramienta era más 
utilizada en los predios que cuentan con un asesoramiento veterinario de forma permanente $(78,2 \pm 7,2 \%)$ que cuando era de tipo eventual $(34,9 \pm 8,4 \%)$. Por otro lado, sólo el 22,8 $\pm 4,8 \%$ contestó vacunar contra todas las enfermedades reproductivas (Brucelosis, Leptospirosis, Diarrea Viral Bovina (DVB), Rinotraqueitis Infecciosa Bovina (IBR) y Campylobacteriosis), sin encontrarse asociación con el tamaño del predio ni con el tipo de asesoramiento veterinario. Dentro de las vacunas utilizadas se reportó que el 40,0 $\pm 5,7 \%$ de los predios vacunaban contra Brucelosis, el 49,0 $\pm 5,4 \%$ contra Leptospirosis, el 32,1 $\pm 5,4 \%$ contra DVB, el 38,3 $\pm 5,4 \%$ contra IBR y el 26,8 $\pm 4,9 \%$ contra Campylobacteriosis. Se encontró una asociación positiva entre la vacunación contra Leptospirosis $(p<0,01)$ e IBR $(p=0,02)$ y el estrato de población bovina. La vacunación para IBR se utilizaba más en los estratos con $>250$ bovinos $(62,5 \pm 8,7 \%)$ que en los dos estratos con $<250$ bovinos donde la proporción fue la misma $(30,0 \pm 8,5 \%)$. De la misma manera para Leptospirosis, la vacuna era más utilizada por los productores más grandes $(87,5$ $\pm 5,9 \%$ ) que por los medianos y pequeños productores $(33,3$ $\pm 8,9 \%$ y $36,7 \pm 8,8$, respectivamente).

\section{Características sanitarias}

Para evaluar los AER se tuvieron en cuenta las siguientes enfermedades: Brucelosis, Leptospirosis, Neosporosis, IBR, DVB, Campylobacteriosis y Trichomoniasis. El 58,7 $\pm 6,7$ $\%$ de los encuestados declaró no tener ningún AER en el predio. Dentro de los predios con AER (41,3\%) se encontraron diferencias significativas entre estratos de población bovina $(p<0,01)$. Los encuestados del estrato con 51-250 bovinos reportaron tener menos AER $(22,2 \pm 10,1 \%)$ que el de $>250$ bovinos $(78,9 \pm 9,6$ $\%$ ). La distribución de cada una de las enfermedades reproductivas se representa en la Tabla 5. Para el caso de Leptospirosis, IBR y Brucelosis se encontraron diferencias significativas con los estratos de población bovina (Tabla 5).

Un $90,9 \pm 4,2 \%$ de los productores lecheros declararon observar abortos el año anterior, no habiendo diferencias significativas entre estratos. Dentro de estos se reportó que la media de abortos vistos anualmente fue de $4,4 \pm 0,6$, siendo mayor este número en el estrato $>250$ bovinos que en los dos estratos restantes $(1,2 \pm 0,4$ vs. $9,0 \pm 1,7)(\mathrm{p}<0,01)$, solo el $16,7 \pm 4,9 \%$ observaron un aumento de estos con respecto a años anteriores. Hubo una asociación positiva entre el número de abortos observados y la presencia de AER, en predios sin antecedentes el número de abortos fue de 2,2 $\pm 0,4$ abortos, mientras que los que tienen antecedentes fue de $8,3 \pm 1,9$.

En la Tabla 6 se representan las causas presumibles de abortos, el nacimiento de animales débiles o muertos, la observación de ciclos largos y la repetición de celo en las vacas en estación reproductiva. Se observa que en la mayoría de los predios los encuestados desconocían la causa de aborto, no habiendo diferencias significativas entre estratos. Se estimó que el nacimiento de animales débiles o muertos, era mayor en estratos de mayor tamaño ( $>250$ bovinos) que en aquellos con 1-50 bovinos $(\mathrm{p}<0,01)(57,1 \pm 8,5 \%$ vs $16,7 \pm 6,9 \%)$. Para las variables restantes no hubo asociación estadística con el tamaño del predio. Tampoco hubo asociación entre la presencia de repetición de celos u observación de ciclos largos con AER.

Tabla 5. Antecedentes de enfermedades reproductivas según encuesta realizada a productores en predios lecheros del Uruguay (Media \pm Error estándar).

\begin{tabular}{llllll}
\hline & Total $(\%)$ & $1-50$ bovinos $(\%)$ & $51-250$ bovinos $(\%)$ & $>250$ bovinos $(\%)$ & Valor-p \\
\cline { 2 - 6 } Leptospirosis & $31,2( \pm 6,2)$ & $23,1( \pm 12,2)$ & $16,7( \pm 9,0)$ & $65,0( \pm 10,9)$ & $<0,01$ \\
Neosporosis & $16,5( \pm 4,8)$ & $23,1( \pm 12,2)$ & $5,6( \pm 5,6)$ & $35,0( \pm 10,9)$ & 0,07 \\
Trichomoniasis & $4,5( \pm 4,3)$ & 0 & $5,6( \pm 5,6)$ & $5,0( \pm 5,0)$ & 0,78 \\
IBR & $4,2( \pm 2,3)$ & 0 & 0 & $15,0( \pm 8,2)$ & $<0,01$ \\
Campylobactoriosis & $4,0( \pm 2,3)$ & $7,7( \pm 7,7)$ & 0 & $10,0( \pm 6,9)$ & 0,13 \\
Brucelosis & $1,2( \pm 1,2)$ & $7,7( \pm 7,7)$ & 0 & 0 & 0,03 \\
DVB & 0 & - & - & - & - \\
\hline
\end{tabular}

Tabla 6. Características sanitarias de predios lecheros del Uruguay.

\begin{tabular}{cll}
\hline Eventos Sanitarios & Media (\%) & Error estándar (\%) \\
\hline Aborto, por causas: & & \\
Desconocidas & 57,4 & 6,7 \\
Nutricionales & 10,0 & 4,2 \\
Venéreas & 1,0 & 1,0 \\
Leptospirosis & 9,4 & 3,4 \\
Brucelosis & 4,3 & 2,7 \\
Otras causas & 12,0 & 4,4 \\
Nacimiento de animales débiles & 33,6 & 5,2 \\
Repetición de celos & 56,5 & 5,6 \\
Ciclos más largos & 26,3 & 5,2 \\
\hline
\end{tabular}




\section{Discusión}

La tasa de respuesta de la encuesta fue alta, este parámetro puede estar influenciado por la estrategia de recolección de datos. En este estudio la encuesta fue de tipo presencial, donde los encargados de realizarla fueron veterinarios. Existen trabajos que reportan tasas de respuesta menores, donde las estrategias fueron otras, vía email, online o por teléfono, este aspecto debe ser tomado en cuenta en el análisis de los datos y en la evaluación de sesgos (Denis-Robichaud et al., 2016; Klein-Jöbstl et al., 2015; Van Schyndel et al., 2019). Para controlar los sesgos de selección, se categorizaron los predios en tres estratos, cada estrato fue calculado y representado por un número de establecimientos adecuado para la proyección posterior de los resultados a la población general.

El tipo de asesoramiento veterinario estuvo fuertemente vinculado al tamaño de los predios. El porcentaje de predios con asesoramiento veterinario permanente aumentaba cuando aumentaba el tamaño poblacional del estrato, interpretando este resultado como una característica asociada a los aspectos demográficos y económicos de los predios de mayor tamaño. Sin embargo, los datos no permitieron evidenciar una diferencia en las tasas de preñez respecto al tipo de asistencia veterinaria. Astaíza et al, (2017) también evaluaron esta característica, y si bien reportaron niveles de asistencia veterinaria menores, concluyeron que la asistencia técnica es considerada como uno de los procesos clave para orientar el desarrollo productivo y tecnológico del sector agropecuario.

Cuando se preguntó por la utilización de diferentes estrategias reproductivas, la técnica de IA fue un procedimiento ampliamente utilizado en predios lecheros uruguayos $(83,8 \pm 5,2$ $\%)$. Si comparamos estos datos con los resultados aportados por la encuesta de INALE (2014), la discrepancia más marcada se ubica en los predios de menor tamaño poblacional o los tambos con menor producción anual de leche, donde INALE reporta tasas de IA menores (28 a 48 \%). Es importante recalcar que los estratos de ambas encuestas tuvieron una construcción distinta, este hecho pudo impactar en las diferencias observadas entre ambos estudios. En nuestro trabajo, aunque no hubo diferencias significativas entre estratos, existe una tendencia a que los predios con mayor tamaño poblacional inseminen más respecto a los de menor tamaño y ambas encuestan acuerdan en este punto. En el año 2001 y 2003 otras encuestas realizadas en predios lecheros uruguayos, arrojaron datos sobre uso de IA de 67,9 \% y $65 \%$ respectivamente (Ibarra et al., 2002; Zaffaroni et al., 2003). A nivel internacional la utilización de IA se estimó entre el 81,1 y el 89,3 \% (Caraviello et al., 2006; NAHMS, 2014; Van Schyndel et al., 2019). Si se comparan los datos actuales con los antecedentes nacionales publicados ya hace más de una década, se observa una evolución positiva en la incorporación de esta tecnología, observándose con mayor énfasis en los predios más grandes y acercándose a porcentajes internacionales como los ya previamente citados.
Dentro de las estrategias de IA, la IA a celo visto fue la más utilizada y distribuida homogéneamente entre los tres estratos. Si se compara este resultado con los datos de la encuesta de "National Dairy Study" de Canadá, se observa que la IA a celo visto fue más utilizada en los establecimientos chicos $(<39$ vacas) y medianos (39 y 85 vacas) respecto a los grandes ( $>85$ vacas). Los autores atribuyen esta diferencia al tiempo insumido de trabajo que requiere esta estrategia, siendo demasiado en tamaños poblacionales grandes lo que hace necesario la incorporación de tecnologías más avanzadas (Van Schyndel et al., 2019). De hecho, en este trabajo la IATF fue utilizada en mayor medida por los pedios más grandes $(15,9 \%)$, pudiendo estar relacionado no solo al tiempo insumido, sino también a los costos que implica la incorporación de este tipo de tecnología. En la presente encuesta, los predios que utilizan los programas de IATF tienen una diferencia de más de $10 \%$ si se los compara con datos de EEUU, donde reportan la utilización de este programa en un 28,0\% de los predios lecheros (NAHMS, 2014).

Los datos que derivaron de la encuesta no permitieron asociar el uso de tecnologías reproductivas con la mejora en las tasas de preñez. Si bien este resultado concuerda con los reportados por Denis-Robichaud et al. (2016), quienes sí lo asocian a las tasas de inseminación más altas, pero no diferencias significativas en la tasa de preñez, consideramos que la ausencia de dicha asociación tampoco descarta este hecho en sí mismo. Para profundizar en este tema se debería de tener en cuenta otros datos, que no fueron consultados por esta encuesta como ser: tipos de protocolos, capacitación del personal, estado de las vacas, etc. (Cavestany, 2000, De Nava, 2011). De Nava (2009), enfatiza que un programa de IATF depende en gran medida de veterinarios especializados con una buena planificación de los protocolos.

El uso de DEP, manejo de condición corporal en vacas, así como manejo diferencial de vaquillonas se aplica mayoritariamente en el estrato de $>250$ bovinos, lo que supone que estas prácticas también se asocian con el tamaño poblacional y con una mayor disponibilidad de recursos tanto técnicos como económicos por parte de estos tambos. Nuevamente el hecho de que la demografía pueda estar directamente asociadas con la implementación de ciertas prácticas de manejo reproductivo ya fue previamente reportado (Denis-Robichaud et al., 2016).

Existen varios métodos disponibles para realizar el diagnostico de gestación en bovinos, como ser: observación visual (retorno al estro), tacto rectal y ecografía (Fricke et al., 2016; Wisnicky et al., 1948). Si bien el diagnóstico de gestación es una práctica que es habitual en los predios lecheros del Uruguay, el uso de ecografía como método diagnóstico es más frecuente en los predios grandes ( $>250$ bovinos). Este resultado podría tener una asociación con el hecho que los predios más grandes tienen un porcentaje de asistencia técnica permanente mayor que el resto. La encuesta de INALE (2014) reportan un porcentaje de diagnóstico de gestación de $46 \%$ (remitentes y queseros), y si se tienen en cuenta solo los remitentes alcanzan un porcentaje mayor, $56 \%$., el cual se acercaría más a nuestro 
reporte. INALE no discrimina entre los que realizan tacto rectal y ecografía. Por otro lado, se debe mencionar, el trabajo de Zaffaroni et al., (2003), quienes realizaron una encuesta a 53 tambos de la cuenca sur del Uruguay, reportando que el $92 \%$ de los predios realizaban algún diagnóstico de preñez, de los cuales el $84 \%$ lo hacían por tacto rectal y el $8 \%$ por ecografía. Consideramos que las diferencias en el diagnóstico de preñez entre nuestra encuesta, la de INALE (2014) y la de Zaffaroni et al., (2003) pueden atribuirse a la diferencia de los marcos geográficos y de muestreo entre estos trabajos. Si bien en nuestra encuesta no se consultó directamente sobre el uso de tacto rectal, se podría asumir que aquellos predios que no realizan ecografía para el diagnóstico, lo hacen a través del tacto $(86,3 \%)$. Como era previsible teniendo en cuenta el tiempo transcurrido entre el trabajo de Zaffaroni et al., (2003) y el nuestro, se encontró un mayor uso de la ecografía como método diagnóstico (13,7 \% vs. $8 \%$ ). Si se compara nuestros datos con los datos de encuestas realizadas en Canadá y EEUU, ambas también reportan el uso de tacto rectal como principal método para el diagnóstico de gestación. La diferencia con estos dos países radica en los porcentajes de uso de ecografía, siendo menor su uso en Uruguay. De todas maneras, tanto en Uruguay como en los reportes internacionales el uso de la ecografía se ve con más frecuencia en predios grandes (NAHMS, 2014; Van Schyndel et al., 2019).

Existen antecedentes de encuestas nacionales que reportan tasas de preñez similares a la encontrada en nuestro trabajo. Lemaire et al., (2011), realizaron una encuesta en predios lecheros uruguayos, la muestra estuvo constituida por predios con asesoramiento veterinario y seguimiento reproductivo lo cual hace que los datos tengan un sesgo, a pesar de lo cual, los investigadores encontraron que el porcentaje de vacas preñadas sobre el total de vacas masa fue de 73,6 \%, y sobre vacas ofrecidas el 78,4 \%. Pereira et al., (2017) realizaron un monitoreo de registros sanitarios y reproductivos en 13 predios lecheros ubicados en el departamento de Florida, encontrando un porcentaje de preñez/vaca masa promedio fue $63 \%$ con variaciones entre predios de 52 a $75 \%$, los cuales fueron independientes del tamaño del rodeo. Ibarra et al., (2002) revelaron a partir de una encuesta a productores remitentes a Cooperativa Nacional de Productores de Leche (Conaprole) una tasa de preñez promedio de 67,7 \% y encontraron que este indicador era más problemático a medida que los predios aumentaban en tamaño poblacional, reportando tasas de preñez en predios de menos de 50 vacas de 75 . Por otro lado, la DIEA, MGAP (2014) reporta tasas de preñez en ganado de cría en el rubro cárnico de $76,2 \%$, si bien las poblaciones y rubro no son las mismas este dato es próximo al reportado por nuestra encuesta y los reportes nacionales previamente citados. Sin embargo, a diferencia del trabajo de Ibarra et al., (2002) en nuestro trabajo no hubo diferencias en la tasa de preñez entre estratos

La evaluación de la capacidad física y sanitaria de los toros aparece como la estrategia más utilizada por los encuestados, esto seguramente sea debido a que son las formas de evaluación más económicas y que requieran menor capacitación técnica. Si bien Crowe et al., (2018) reportaron las ventajas de realizar la evaluación de la capacidad de servicio y de la calidad seminal de los toros en los parámetros reproductivos, con los datos recabados en esta encuesta no se pudo detectar el impacto de las mismas en las tasas de preñez, pero si se pudo estimar el bajo porcentaje de establecimientos que lo realizan.

La práctica de mantener al toro durante todo el año con las vacas se da más en tambos de 1-50 bovinos, y este porcentaje disminuye a medida que aumenta el estrato de población bovina. Esta forma de manejo trae como consecuencia que las pariciones en estos predios se desarrollen de forma continua durante todo el año, sugiriendo que en tambos más grandes se busca concentrar las épocas de pariciones para llegar a tener picos de producción de leche y así intensificar sus sistemas productivos.

Las principales causas de refugo son: reproductivas (incapacidad para concebir), mastitis, y baja producción (Ansari-Lari et al., 2012; Bascom et al.,1998). En este trabajo los encuestados destacaron a la dentición como principal causa de refugo, independientemente del estrato de población bovina; la causa reproductiva no resulto la más importante. En esta encuesta no se evaluaron causas productivas como posibles causas de refugo y dentro de las sanitarias no se especificaron cuales podrían estar involucradas.

La administración de vacunas es considerada uno de los aspectos más importantes para el control de enfermedades (Tizard, 2009). En el presente trabajo la práctica de vacunación se vio asociada al tamaño del predio y tipo de asesoramiento veterinario, pero no a los AER. La asociación del uso de vacunas reproductivas con el tamaño del predio también se vio en los resultados de la encuesta de INALE. Da Silva Silveira, (2019) realizó un relevamiento de aspectos sanitarios en 20 tambos, con un mínimo de 60 y un máximo de 750 vacas y con antecedentes de abortos, el $85 \%$ de los establecimientos tenía antecedentes de vacunación contra alguna de las enfermedades abortivas. De los tambos que usaban vacunas, $47 \%$ utilizaban solamente contra Leptospirosis y el $35 \%$ usaban vacunas para más de un agente (DVB, IBR, Campylobacteriosis, Leptospirosis). Si bien el trabajo de Da Silva Silveira, (2019) no fue un muestreo aleatorio, nuestra encuesta acuerda respecto al porcentaje de vacunación contra Leptospirosis $(49,0 \pm 5,4 \%)$ no siendo así el porcentaje de vacunación de otras enfermedades reproductivas. Así mimo, hay que destacar que este hecho también acuerda con trabajos internacionales donde reportan la vacuna contra Leptospirosis como una de las más utilizadas junto con DBV e IBR (Cresswell et al., 2014). Además, Cresswell et al., (2014) destacan el rol del veterinario como el principal proveedor de vacunas y fuente principal de información sobre éstas y reflexiona que muchos de los productores que no usan vacunas es debido a que no logran detectar problemas en sus rodeos. Esto no pudo quedar demostrado en nuestro estudio ya que los antecedentes de vacunación no se asociaron con los AER, aunque hay que mencionar que los datos sobre AER podrían contener un sesgo ya que la encuesta no incluía la confirmación por diagnóstico de laboratorio para establecer con mayor certeza estos antecedentes. 
Estudios nacionales estimaron la seroprevalencia de diferentes enfermedades infecciosas que afectan la reproducción como ser: Leptospirosis, Neosporosis, Campylobacteriosis, Brucelosis, IBR y DBV (da Silva Silveira, 2019; Macchi et al., 2020; De Freitas et al., 2005; Repiso et al., 2005). Debido a estos antecedentes dichas enfermedades fueron consultadas en esta encuesta. La Leptospirosis fue una de las enfermedades que más se reportó sobre todo en predios grandes, lo cual tiene correspondencia con la mayor vacunación en este estrato $(>250$ bovinos).

La media de abortos reportada por esta encuesta está dentro de los porcentajes reportados por otros autores (Hovingh et al., 2009; Norman et al, 2012) y su asociación con la presencia de AER. Este resultado coincide con un estudio nacional realizado por Macías-Rioseco, (2019), quien reportó a partir de un estudio de serie de 102 casos de abortos que el $50 \%$ de los casos estudiados respondían a una causa infecciosa.

\section{Conclusión}

Con el objetivo de generar información sobre las características reproductivas y el manejo sanitario empleado en la cuenca lechera del Uruguay se realizó esta encuesta, la misma tiene la fortaleza de partir de datos con un diseño estadístico representativo de la población de establecimientos lecheros y una alta tasa de respuesta. Al igual que en otros países el análisis de esta encuesta pudo revelar que el aspecto demográfico cumple un rol significativo en la elección de los aspectos reproductivos, sanitarios y de manejo, donde los predios de más de 250 bovinos presentan características distintivas respecto a predios con tamaños menores. Sin embargo, a partir de estos datos no se pudo demostrar que las diferencias en las características reproductivas, sanitarias y/o de manejo tuvieran un impacto en las tasas de preñez. Sin embargo, este trabajo establece antecedentes para futuras investigaciones que estudien la influencia de diferentes indicadores en los resultados productivos y económicos en los predios lecheros del Uruguay.

\section{Agradecimientos}

Los autores agradecen a todos los productores que participaron de la encuesta. A los Veterinarios oficiales de la División de Sanidad Animal, del Ministerio de Ganadería Agricultura y Pesca quienes fueron los encargados de realizar la encuesta. Al Dr. Ricardo Zaffaroni por sus aportes y lectura crítica. A la Agencia Nacional de Investigación e Innovación por la financiación del presente trabajo a través del proyecto FSSA_X_2014_1_105711

\section{Referencias}

Ansari-Lari, M., Mohebbi-Fani, M., y Rowshan-Ghasrodashti, A. (2012). Causes of culling in dairy cows and its relation to age at culling and interval from calving in Shiraz, Southern Iran. Veterinary Research Forum, 3(4), $233 \square 237$.

Astaíza, J.M., Muñoz, M.R., Benavides, C.J., Vallejo, D.A., y Chaves, C.A. (2017). Caracterización técnica y productiva de los sistemas de producción lechera del valle de Sibundoy, Putumayo (Colombia). Revista de Medicina Veterinaria, $34,31-43$.

Bascom, S. S. y Young, A. J. (1998). A summary of the reasons why farmers cull cows. Journal of Dairy Science, 81(8), 2299-2305.

Bauman, C. A., Barkema, H. W., Dubuc, J., Keefe, G. P., y Kelton, D. F. (2016). Identifying management and disease priorities of Canadian dairy industry stakeholders. Journal of Dairy Science, 99(12), 10194-10203.

Belage, E., Dufour, S., Bauman, C., Jones-Bitton, A., y Kelton, D. F. (2017). The Canadian National Dairy Study 2015-Adoption of milking practices in Canadian dairy herds. Journal of Dairy Science, 100(5), 3839-3849.

Caraviello, D. Z., Weigel, K. A., Fricke, P.M, Wiltbank, M.C., Florent, M.J., Cook, N.B., y Rawson, C.L. (2006). Survey of Management Practices on Reproductive Performance of Dairy Cattle on Large US Commercial Farms. Journal of Dairy Science, 89, 4723-4735.

Cavestany, D. (2000). Resumen de ensayos sobre eficiencia reproductiva en vacas de leche en producción, factores que la afectan y alternativas de manejo para incrementarla (Período 1992 a 1997). En Temas de lechería: reproducción. (pp.1-18). Montevideo: INIA.

Cresswell, E., Brennan, M.L., Barkema, H.W., y Wapenaar, W. (2014). A questionnaire-based survey on the uptake and use of cattle vaccines in the UK. Veterinary Record Open, 1(1), 1-10.

Crowe, M.A., Hostens, M., y Opsomer, G. (2018). Reproductive management in dairy cows the future. Irish Veterinary Journal, 71(1), 2-13.

da Silva Silveira, C. (2019). Enfermedades infecciosas que causan abortos en bovinos con enfoque en rodeos lecheros del Uruguay. (Tesis de Doctorado. Universidad de la República). Recuperado de: https://www.colibri. udelar.edu.uy/jspui/bitstream/20.500.12008/24123/1/ silvaS.pdf

De Freitas, J., Gil, A., Piaggio, J., Nuñez, A., Suanes, A., Zaffaroni, R., ... Cernicchiaro, N. (2005). Seroprevalence evolution of infectious bovine rinotraqueitis (IBR) and bovine diarrhea (BVD) in dairy cattle population of the southern region of Uruguay. En $12^{\circ}$ Simposio Internacional de la Asociación Mundial de Laboratorios de Diagnóstico Veterinario (WAVLD) (pp 124). Montevideo: INIA. 
de Nava, G. (2009). El rol del veterinario en la instrumentación de programas de IATF. En XLIII Jornadas Uruguayas de Buiatría (pp. 126-137). Paysandú: Centro Médico Veterinario de Paysandú.

de Nava, G. (2011). Un manejo reproductivo controlado en tambos de Uruguay. En XXXIX Jornadas Uruguayas de Buiatría (pp. 1-23). Paysandú: Centro Médico Veterinario de Paysandú.

Denis-Robichaud, R. L. A., Cerri, A., Jones-Bitton, S.J., y LeBlanc, J. (2016). Survey of reproduction management on Canadian dairy farms. Journal of Dairy Science, 99(11), 9339-9351.

Dirección de Investigaciones Económicas Agropecuarias, Ministerio de Ganadería Agricultura y Pesca (2014). Estadísticas agropecuarias. Instituto Nacional de Carnes. Montevideo, DIEA. Disponible en: https://www.gub. uy/ministerio-ganaderia-agricultura-pesca/comunicacion/publicaciones/anuario-estadistico-diea-2014

Fricke, P.M., Ricci, A., Giordano, J.O., y Carvalho, P.D. (2016). Methods for and implementation of pregnancy diagnosis in dairy cows. Veterinary Clinics of North America: Food Animal Practice, 32, 165-80.

Hovingh, E. (2009). Abortions in dairy cattle: I. Common causes of abortions. Virginia Coop. Ext. Publ 404-288.

Ibarra, D. (2002). Indicadores reproductivos en la cuenca lechera de Conaprole en los servicios de otoño de 2001. En XXX Jornadas Uruguayas de Buiatría (pp. 256-258). Paysandú: Centro Médico Veterinario de Paysandú.

INALE (2019). Situación y perspectiva de la lechería uruguaya. Informes INALE, 18. Recuperado de https://www.inale.org/informes/informe-2019/

INALE (2014). Encuesta Lechera INALE 2014: resultados preliminares. Recuperado de: https://www.inale.org/ historico/wp-content/uploads/2018/08/Encuesta-lechera-2014.pdf

Klein-Jöbstl, D., Arnholdt, T., Sturmlechner, F., Iwersen, M., y Drillich, M. (2015). Results of an online questionnaire to survey calf management practices on dairy cattle breeding farms in Austria and to estimate differences in disease incidences depending on farm structure and management practices. Acta Veterinaria Scandinavica, 57(1), 44-53.

Lemaire, C., Grela, C., De María, P., y Cavestany, D. (2011). Indicadores reproductivos en predios lecheros en Uruguay: Resultados de dos años de evaluación. Veterinaria (Montevideo), 48 (184), 17-22.

Macchi, M. V., Suanes, A., Salaberry, X., Fernández, F., Piaggio, J., y Gil, A.D. (2020). Epidemiological study of neosporosis in Uruguayan dairy herds. Preventive Veterinary Medicine, 179, 1-6.

Macías-Rioseco, M. (2019). Causas de aborto en bovinos lecheros de Uruguay. (Tesis de Doctorado inédita). Universidad de la República, Montevideo, Uruguay.

Norman, H.D., Miller, R.H., Wright, J.R., Hutchison, J.L., y Olson, K.M. (2012). Factors associated with frequency of abortions recorded through Dairy Herd Improve- ment test plans. Journal of Dairy Science, 95(7), $4074 \square 4084$.

OPYPA (2019). Oficina de Programación y Politica Agropecuaria. Recuperado de https://descargas.mgap.gub.uy/ OPYPA/Anuarios/Anuario\%202019/ORIGINAL\%20 2019\%20OPYPA\%20INTERACTIVO\%20agregado\%2018-12-2019.pdf

Pereira, I., Cruz, I., Ruprechter, G., y Meikle, A. (2017). Salud y eficiencia reproductiva de vacas lecheras en sistemas de base pastoril de Florida: resultados preliminares del monitoreo. En XLV Jornadas Uruguayas de Buiatría (pp. 127-139). Paysandú: Centro Médico Veterinario de Paysandú.

Rao, J.N.K., y Scott, A.J. (1984). On chi-squared tests for multiway contingency tables with cell proportions estimated from survey data. Annals of Statistics, 12, 46-60.

Renaud, D. L., Duffield, T. F., LeBlanc, S. J., Haley, D. B., y Kelton, D. F. (2017). Management practices for male calves on Canadian dairy farms. Journal of Dairy Science, 100(8), 6862-6871.

Repiso, M.V., Gil, A., Bañales, P., D’Anatro, N., Fernández, L., Guarino, H., ... Silva M. (2005). Prevalencia de las principales enfermedades infecciosas que afectan el comportamiento reproductivo en la ganadería de carne y caracterización de los establecimientos de cría del Uruguay. Veterinaria, 40, 5 -28.

Tizard, I. R. (2009). Veterinary immunology: an introduction. $\left(8^{\mathrm{a}}\right.$ ed). Texas: Saunders.

United States Department of Agriculture Animal and Plant Health. Inspection Service Veterinary Services. National Animal Health Monitoring System. (2018). Report 3: Dairy 2014. Health and Management Practices on U.S. Dairy Operations. Recuperado de https:// www.aphis.usda.gov/animal_health/nahms/dairy/ downloads/dairy14/Dairy14_dr_PartIII.pdf

Van Schyndel, S. J., Bauman, C. A., Pascottini, O. B., Renaud, D. L., Dubuc, J. y Kelton, D. F. (2019). Reproductive management practices on dairy farms: The Canadian National Dairy Study 2015. Journal of Dairy Science, 102(2), 1822-1831.

Wisnicky, W. y Cassida, L.E. (1948). A manual method for diagnosis of pregnancy in cattle. Journal of the American Veterinary Medical Association, 113, 451452.

Zaffaroni, R., Cernicchiaro, N., De Freitas, J., Piaggio, J., Suanes, A., Huertas, S., y Gil, A. (2003). Encuesta sobre características reproductivas y de manejo en establecimientos lecheros del Uruguay. En XXXI Jornadas Uruguayas de Buiatría (pp. 129). Paysandú: Centro Médico Veterinario de Paysandú.

\section{Notas de contribución:}

Concepción y diseño del estudio, 2. Adquisición de datos, 3. Análisis de datos, 4. Discusión de los resultados, 5. Redacción 
del manuscrito, 6. Aprobación de la versión final del manuscrito.

Alejandra Suanes ha contribuido en 1, 2, 3, 4, 5 y 6. Valentina Macchi ha contribuido en 1, 2, 3 4, 5 y 6. Federico Fernández ha contribuido en 1, 2, 3, 4, y 6. Ximena Salaberry ha contribuido en 1, 2 y 6. Cyntia Moreira ha contribuido en 1, 2 y 6. Andrés Gil ha contribuido en $1,2,3,4, \mathrm{y} 6$.

El editor Cecilia Cajarville aprobó este artículo.

Anexo I

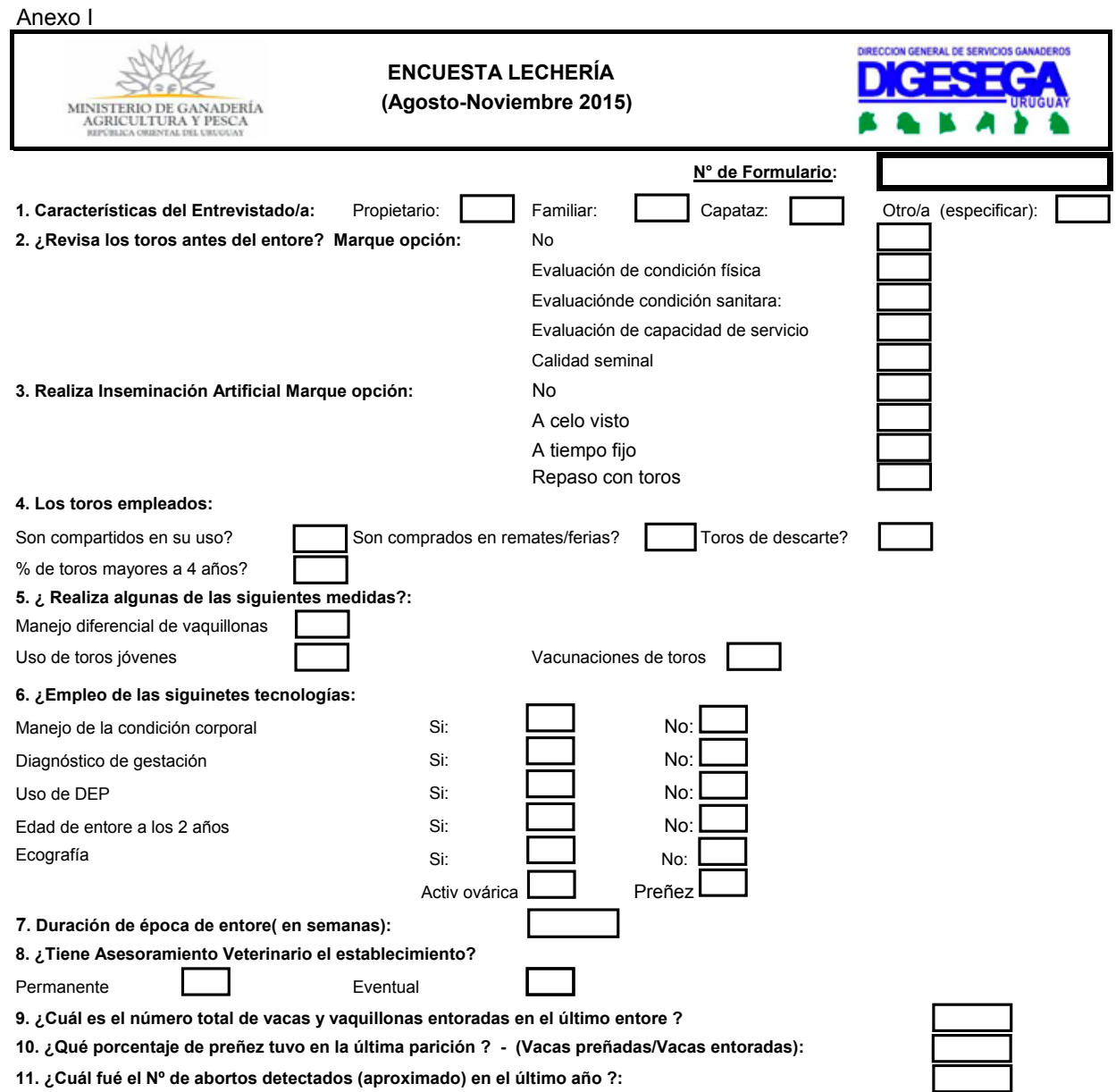

12. ¿Causa atribuible de abortos?

Nutricionales: $\square$ Venéras $\square$ Leptospira $\square$ Brucelosis $\square$ Otras $\square$ Desconcoce

13. Por los antecedentes del establecimiento, ¿el \% de abortos es mayor que en años anteriores?(SI/NO):

14. ¿Hubo nacimientos de animales débiles o muertos? (SI/NO):

15.Durante el entore, observó; Repetición de celo?

16.Causas de refugo de hembras: Dentición ( $\mathrm{SI} / \mathrm{NO}$ ):

Falla por 1a. vez (SI/NO)

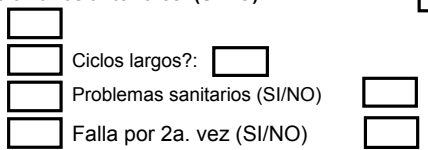

17.Hay antecedentes de enfermedades reproductivas en el predio?:

No Neosporosis

Leptospirosis

BVD

Campilobacteriosis

Tricomoniasis

Brucelosis

IBR

18. Realiza vacunaciones contra:
Brucelosis

Leptospirosis

BVD

IBR

Campilobacteriosis
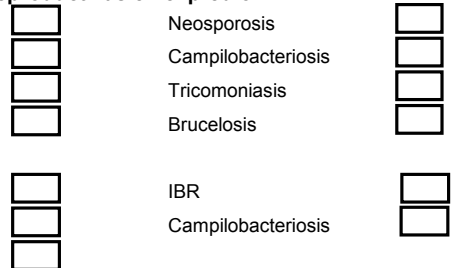